changes. Our objective is to report a case of Rhodococcus pneumonia in a HIV patient.

Methods 29 year old male, from Rio de Janeiro, cocaine user. Presented in Mar/16 with productive cough and hemoptysis, high fever, weight loss, sweating, right pleuritic pain and dyspnea. In Apr/16, he was treated for bacterial pneumonia. Symptoms persisted and by the end of May/16, pulmonary TB was suspected. AFB were detected in sputum. GeneXpert test was negative. Chest $\mathrm{x}$-ray showed multiple cavitations in right upper lober. In June/16 RHZE was started. Three days later, he presented with abdominal pain, vomiting and diarrhoea and was hospitalised. RHZE was suspended and HIV diagnosis was made at this time. On admission, he was febrile $\left(38.1^{\circ} \mathrm{C}\right)$, tachypneic and pale; crackling rales in the upper $1 / 3$ of the right lung. Liver function tests were normal. RHZE was reintroduced. FBC showed mild anaemia and leukocytosis; normal renal function. Chest $\mathrm{x}$-ray revealed abscesses with thickened walls and an air-fluid level, and extensive consolidation in RUL. Because of the dissociation between sputum smear microscopy and GeneXpert results, rhodococcus infection was suspected and levofloxacin was started. Blood cultures showed growth in 3 samples and Gram staine was suggestive of corynebacteria. Rhodococcus was identified by Coryne API. Chest CT showed ground-glass infiltrate, and sulfamethoxazole-trimethoprim for PCP was initiated. The patient was afebrile after 72 hours of levofloxacin and rifampicin. $\mathrm{He}$ was discharged and is currently well, followed at the outpatient unit.

Discussion and conclusion The case was suggestive of TB but was confirmed as Rhodococcus. Because it is rare, it can lead to delayed diagnosis, inadequate therapy and increased mortality. It is important to investigate rodhococcosis in HIV patients with TB criteria, especially in case of dissociation between smear microscopy and GeneXpert.

\section{P2.26 EXOTIC MYCOBACTERIA IN A PAKAREN MACHADO GOMESIENT WITH HIV IMMUNOSUPPRESSION}

${ }^{1}$ Marcos Davi Gomes de Sousa, ${ }^{2}$ Karen Machado Gomes, ${ }^{1}$ Maria Cristina da Silva Lourenco, ${ }^{1}$ Cristiane Lamas. 'National Institute of Infectology Evandro Chagas, Rio de Janeiro - RJ, Brazil, ${ }^{2}$ Federal University of Rio De Janeiro, Rio de Janeiro - RJ, Brazil

10.1136/sextrans-2017-053264.202

Introduction: Mycobaterium colombiense, a slow-growing mycobacterium, belongs to the complex of Mycobaterium avium (MAC). It was isolated for the first time in Colombia, in 2006, in an HIV patient. To report the first case of a brazilian HIV/AIDS patient in whom M. colombiense was identified.

Methods 51 year old male, heavy drinker and smoker, COPD, waste picker. HIV+, poorly adherent to ART, CD4 count of 14 and VL 3,960 in 2015. He was treated for M. kansasii infection from 2007 to 2009, when ART was also started. In 2015, he had several sputa collected, and results were intermittently positive for AFB testing. GeneXpert was negative in all of samples. He had a positive culture for M. intracellulare in January; but sputum culture was positive for atypical mycobacteria in September and November. BAL in November had a positive culture for MAC, later identified as M. colombiense. The clinical specimen was treated by the NALC-NaOH method, and was seeded in LJ medium. Growth of smooth, creamy colonies with yellow pigment was observed after 30 days of incubation. Sequencing of the rpoB gene for identification showed similarity of $99 \%$ with the CIP 108962 strain M. colombiense. The gene sequence was analysed by BLAST V2.0. All blood cultures for fungus, mycobacteria and bacteria were negative in the same time.

Discussion and conclusion This patient with advanced AIDS, COPD and alcoholism had been treated years before for $M$. kansasii and for M. avium. Six years later, due to respiratory and consumptive syndrome, he was hospitalised for investigation and there was growth of $M$. colombiense in bronchoalveolar lavage - BAL. To our knowledge, this is the first isolate of this species in Brazil. In 2015, M. intracellulare and MAC were grown in sputum culture; these mycobacteria, being closely related, are very difficult to distinguish, with the possibility that $M$. colombiense was the etiological agent early on. Due to the unavailability of modern moleculares tools to describe emerging MAC species, the true prevalence of $M$. colombiense in Brazil is probably underestimated.

\section{P2.27 HIGH SATISFACTION WITH AND LOYALTY TO GETCHECKEDONLINE.COM AMONG FIRST-TIME USERS OF AN ONLINE STI TESTING SERVICE IN BRITISH COLUMBIA, CANADA}

${ }^{1}$ Mark Gilbert, ${ }^{1}$ Kimberly Thomson, ${ }^{1}$ Travis Salway, ${ }^{1}$ Devon Haag, ${ }^{1}$ Troy Grennan, ${ }^{2}$ Chris Buchner, ${ }^{1}$ Mark Tyndall, ${ }^{1}$ Mel Krajden, ${ }^{1}$ Gina Ogilvie, ${ }^{3}$ Jean Shoveller. ${ }^{1}$ British Columbia Centre for Disease Control, Vancouver, Canada; ${ }^{2}$ Fraser Health Authority, Vancouver, Canada; ${ }^{3}$ University of British Columbia, Vancouver, Canada

\subsection{6/sextrans-2017-053264.203}

Introduction Positive user experiences are key to trust and repeated use of online services (known as e-Loyalty). GetCheckedOnline (GCO) is an online testing service for HIV/STI where clients complete a risk assessment, print lab forms, submit specimens at a lab, and retrieve results online (if negative) or by phone. We surveyed GCO clients on their perceptions of using the service.

Methods We invited first-time GCO users (who consented to be contacted for research) to complete an anonymous online survey 2 weeks following reporting of test results. Survey questions were analysed descriptively and included demographics, reason for test, and how participants heard about GCO. Satisfaction, convenience, ease of use, and e-Loyalty (intention to use again, recommend to others) were measured using 5-point Likert scales and collapsed (low to neutral vs high responses).

Results Between July 2015-Sept 2016, 23\% of 1099 first-time GCO users consented to be contacted for research and 136/ $208(65 \%)$ of users contacted agreed to participate in the survey. Participants had a median age of 33 years, $80 \%$ were white, $67 \%$ male, $43 \%$ straight, and $43 \%$ men who have sex with men. The most common testing reasons were: routine test (64\%), risk event/exposure (44\%) and new relationship (22\%). Participants heard about GCO from clinics/health providers (38\%), campaigns (26\%), social media (18\%), and friends or partners $(13 \%)$. Almost all participants were satisfied with GCO overall (93\%) and with their experience of receiving results (96\%), 92\% agreed GCO was convenient, $87 \%$ found GCO easy to use, and $83 \%$ rated the experience of submitting specimens as good or excellent. E-Loyalty was also high: $97 \%$ intended to use GCO again and 96\% would recommend GCO to others. 
Conclusion We found very high satisfaction with and loyalty to GCO among first-time users, indicating a successful service model from a client perspective. In addition to uptake and test outcomes, user experience is a key outcome for evaluation of online HIV/STI testing services.

\section{P2.28 ART - INDUCED NEPHROTOXICITY AND CHRONIC KIDNEY DISEASES AMONG AMBULATORY HIV - INFECTED PATIENTS WITH LOW BODY MASS INDEX IN BRAZZAVILLE, CONGO: INCIDENCE AND ASSOCIATED RISK FACTORS}

Martin Herbas Ekat. University Hospital of Brazzaville

10.1136/sextrans-2017-053264.204

Introduction To describe the incidence and risks factors of ART induced nephrotoxicity and chronic kidney disease (CKD) in HIV-1-infected adults with low body mass index $\left(<18.5 \mathrm{~kg} / \mathrm{m}^{2}\right)$.

Methods A retrospective cohort study at the Ambulatory Treatment Centre in Brazzaville, Congo. Patients with estimated glomerular filtration rate (eGFR) decrease by $25 \%$ compared to baseline or a $0.5 \mathrm{mg} / \mathrm{dL}$ increase in Serum creatinine ( $\mathrm{Scr}$ ) above baseline were classified as having nephrotoxicity, and $\mathrm{CKD}$ was defined as a value less than $60 \mathrm{ml} / \mathrm{min}$ per $1.73 \mathrm{~m}^{2}$. We used Cox proportional hazards regression models to determine factors associated with nephrotoxicity and CKD. Results Of 325 patients, $73.23 \%$ were women. Median values was: age: 37.55 years (IQR: 33.51-44.96), weight: $45 \mathrm{~kg}$ (IQR: 41-49), CD4 count: 137.5 cells/ $\mu 1$ (42 - 245). In the first 24 - months followup on ART incidence rate of nephrotoxicity and CKD was 27.95 and 7.44 per 100 person - years respectively. Multivariate analysis identified as a risk factor of nephrotoxicity, baseline haemoglobin below or equal $8 \mathrm{~g} / \mathrm{dL}$ $(\mathrm{aHR}=2.25 ; 95 \% \mathrm{CI}, 1.28-3.98 ; \mathrm{p}=0.005)$, eGFR between $60-80(\mathrm{aHR}=0.33 ; 95 \% \mathrm{CI}, 0.20-0.56 ; \mathrm{p}=0.001)$ and below $60 \mathrm{ml} / \mathrm{min} / 1.73 \mathrm{~m}^{2} \quad(\mathrm{aHR}=0.11 ; 95 \% \mathrm{CI}, 0.03-0.46 ; \mathrm{p}=0.003)$, and the use of tenofovir $(\mathrm{aHR}=1.51 ; 95 \% \mathrm{CI}, 1.01-2.26$; $\mathrm{p}=0.04)$. Each 10 year older age was associated with an increased risk of developing CKD $(\mathrm{aHR}=1.95$; 95\% CI, 1.2$3.17 ; \mathrm{p}=0.007)$.

Conclusion Incidence of nephrotoxicity and CKD were high. HIV positive patient with low BMI at baseline need close monitoring of their renal function when treated with tenofovir.

\section{P2.29 PERFORMANCE OF A CLINICAL PREDICTION SCORE FOR TARGETED CREATININE TESTING IN AFRICA}

Martin Herbas Ekat. University Hospital of Brazzaville, Republic of Congo

\subsection{6/sextrans-2017-053264.205}

Introduction in sub-saharian africa, in clinical practice only patients meeting the WHO criteria to start antiretroviral treatment undergone creatinine testing, by lack of reagents. Some patients with renal dysfunction are not diagnosed. Van Griensven et al. developed a clinical prediction score (CPS), to accurately identify Cambodian patients, with median baseline body weight of $49 \mathrm{~kg}$ (IQR: 43-55), who need a creatinine test before initiating antiretroviral therapy, based on the post-test probability of renal dysfunction. We evaluated the performance of this CPS for predicting patients at risk of kidney dysfunction (KD) in Brazzaville, Congo.

Methods The CPS includes following predictors: age (score +2 if $>40$ years), body weight (score +2 if $<45 \mathrm{~kg}$ ), and haemoglobin (score +1 if $>10 \mathrm{~g} / \mathrm{dL}$ ), the alternative CPS includes: age (score +2 if $>40$ years), body weight (score +2 if $<45 \mathrm{~kg}$ ), sex (score +1 if female), and WHO stage (score +1 if WHO stage III/IV). The overall test performance of the CPS was assessed by calculating the area under the receiver-operating characteristic (AUROC) curve. We defined KD as an estimated creatinine clearance based on the Cockcroft - Gault equation, by using two threshold, $<50$ (CG1) and $<60$ (CG2) $\mathrm{mL} / \mathrm{min}$. Results Among 545 patients, median values was: body weight $55 \mathrm{~kg}$ (IQR 48-63), age 38.87 years (33.18-46.21); 76 $(13.95 \%)$ and $142(26.06 \%)$ patients, respectively for CG1 and CG2, had KD. AUROC was 0.6183 (CPS) and 0.5815 (alternative CPS) for CG1 ( $\mathrm{p}=0.0541), 0.7140$ (CPS) and 0.6691 (alternative CPS) for CG2 $(\mathrm{p}=0.0016)$.

Conclusion Because the baseline body weight of African is high than in Cambodian patients, the CPS must be designed for African patients, it is a useful supplement to clinical judgment in the era of limited resources.

\section{P2.30 SURVEY OF ANTIMICROBIAL RESISTANCE IN CLINICAL NEISSERIA GONORRHOEAE ISOLATED OVER A PERIOD OF FOUR YEARS IN NAIROBI - KENYA}

${ }^{1}$ Meshack Juma Omolo, ${ }^{1}$ Lewa Pole, ${ }^{1}$ Isabella Mwangi, ${ }^{2}$ Joseph Kimani, ${ }^{1}$ Omu Anzala ${ }^{3}$ Jonathan Oloo, Wi, ${ }^{4}$ Teodora Elvira, ${ }^{5}$ Susanne Jacobsson, ${ }^{6}$ Magnus Unemo. ${ }^{1}$ Kavi-Institute of Clinical Research (UON), Nairobi, Kenya; ' 2 Sex Workers Outreach Program Clinic (SWOP), Nairobi, Kenya; ${ }^{3}$ Dept Medical Microbiology (UON), Nairobi, Kenya; ${ }^{4}$ World Health Organisation (WHO), Geneva, Switzerland; ${ }^{5}$ WORLD WHO Collaboration Centre for Gonorrhoea and Other ST/S Orebro University, Örebro, Sweden; ${ }^{6}$ WORLD WHO Collaboration Centre for Gonorrhoea and Other STIs Örebro University, Örebro, Sweden

\subsection{6/sextrans-2017-053264.206}

Introduction There is increasing urgency to document changing antimicrobial resistance (AMR) patterns of $N$. gonorrhoea (GC) in different parts of the world. High-level resistance to previously recommended quinolones is widespread and decreased susceptibility to the extended-spectrum (third-generation) cephalosporin. The surveillance for AMR in Kenya and the region was undertaken to determine the frequency and diversity of antimicrobial resistance of gonococcal isolates from Sex Workers Outreach Program (SWOP) Clinic.

Methods The survey tested 238 isolates over a period of 4 years from participants presenting with cervical/vaginal discharge. Samples collected were inoculated directly on modified Thayer martin media (MTM), transported to GASP Laboratories at KAVI-Institute of Clinical Research and identified by standard bacteriological procedures. Antibiotic susceptibility testing of $G C$ isolates was performed using diffusion gradient method. The MICs of penicillin, tetracycline, ciprofloxacin, spectinomycin, erythromycin, Azithromycin, cefixime and ceftriaxone were determined by the E-test method. The strains were defined as susceptible, intermediate and resistant using the WHO guidelines, all the findings were validates at WHO Collaborating Centre for Gonorrhoea and other STIs, Örebro University Hospital in Sweden.

Results 41 isolates in 2012,119 isolates in 2013, 24 isolates in 2014 and 54 isolates in 2015 showed 100\% susceptaility for cefixime, ceftriaxone and spectinomycin, with a mean susceptibility of $82 \%, 37.7 \%, 19.5 \%, 1.6 \%$ and $0 \%$ for azithromycin, 